\title{
Wege zur Gestaltung agiler Klimaanpassungsprojekte
}

\author{
Transformative und generalisierbare Lösungsansätze für Kommunen
}

\author{
Simone Häußler ${ }^{1}(\mathbb{D}) \cdot$ Martina Hofmann ${ }^{1} \cdot$ Martin Müller $^{2}$ \\ Eingegangen: 27. Dezember 2020 / Überarbeitet: 9. März 2021 / Angenommen: 12. März 2021 / Online publiziert: 9. April 2021 \\ (c) Der/die Autor(en) 2021
}

\section{Zusammenfassung}

Die Auswirkungen des Klimawandels stellen Städte vor große Herausforderungen. Sie sind besonders vulnerabel für Schäden infolge von Extremwetterereignissen. Daher besteht eine besondere Dringlichkeit, wirkungsvolle Klimaanpassungsmaßnahmen in den Städten zu initiieren. Die Umsetzung und die Verstetigung dieser Maßnahmen bleiben bis dato häufig hinter dem Notwendigen zurück. Es besteht die Gefahr, dass wichtige Klimaanpassungsprojekte möglicherweise zu spät oder gar nicht umgesetzt werden und dadurch ihre Wirkung nur bestenfalls unzureichend entfalten können. Das Ziel dieser Arbeit ist, die Ursachen dieser Hemmnisse am Beispiel Baden-Württemberg aufzudecken, um agiles und fokussiertes Handeln im kommunalen Bereich zu den drängenden Fragen der Klimaanpassung zu verbessern. Es geht um die Beantwortung der Frage: Wo liegen die Hemmnisse für die effektive Integration von Klimaanpassungsmaßnahmen in Kommunen? Und darauf aufbauend: Welche Lösungsansätze tragen zur Überwindung dieser Hemmnisse bei? Hierzu wurde mit Fachund Führungskräften der Bereiche Klimaschutz und Klimaanpassung aus baden-württembergischen Kommunen ein zweitägiger Workshop durchgeführt. Die entwickelten Lösungsvorschläge zur Überwindung der Hemmnisse in den Bereichen Politik, Kommunikation und Organisation sind im Wesentlichen: die Schaffung lokaler Interessensvertretungen in Form von Netzwerken; die Etablierung strategischer Stakeholder-Kooperationen für Wissenstransfers sowie Veränderungen in den kommunalen Prozessen und Organisationsstrukturen.

\section{Schlüsselwörter Baden-Württemberg $\cdot$ Städte $\cdot$ Klimawandel $\cdot$ Netzwerke $\cdot$ Wissenstransfer}

\begin{abstract}
The impacts of climate change pose major challenges for cities. They are particularly vulnerable to damage as a result of extreme weather events. Therefore, there is a particular urgency to initiate effective climate adaptation measures in cities. To that end, the implementation and continuation of these measures often fall short of what is necessary. There is a risk that important climate adaptation projects may be implemented too late or not at all, and thus their impact may be inadequate at best. The aim of this work is to uncover the causes of these obstacles using the example of Baden-Württemberg in order to improve agile and focused action in the municipal sector on the pressing issues of climate adaptation. The question is, where are the obstacles to the effective integration of climate adaptation measures in municipalities? And, based on this, which problem-solving approaches can help to overcome these obstacles? For this purpose, a two-day workshop was conducted with experts in the fields of climate protection and climate adaptation from municipalities in Baden-Württemberg. The suggested solutions developed to overcome obstacles in the areas of politics, communication and organisation are: the creation of local networks of action, the establishment of strategic stakeholder cooperation for knowledge transfer and changes in the municipal processes and organisational structures.
\end{abstract}

Simone Häußler, M.Sc.

Simone.Haeussler@hs-aalen.de

1 Elektrotechnik - Erneuerbare Energien, Hochschule Aalen Technik und Wirtschaft, Anton-Huber-Str. 25, 73430 Aalen, Deutschland

2 Institut für Nachhaltige Unternehmensführung, Fakultät für Mathematik und Wirtschaftswissenschaften, Universität Ulm, Helmholtzstraße 18, 89081 Ulm, Deutschland 
Keywords Baden-Württemberg $\cdot$ Cities $\cdot$ Climate change $\cdot$ Networks $\cdot$ Knowledge transfer

\section{Einleitung}

Seit dem Rekordsommer im Jahr 2003 häufen sich Extremwetterereignisse von Hitze und Trockenheit in großen Teilen Mitteleuropas. Dabei wurde am Fall Baden-Württembergs deutlich, welche starken Auswirkungen der Klimawandel auch für gemäßigte Klimazonen mit sich bringen kann (Stölzle und Stahl 2011). Die Frequenz und die Intensität der sogenannten Hitzetage, definiert durch eine Tagesmaximaltemperatur $\geq 30^{\circ} \mathrm{C}$, nehmen landesweit zu (Abb. 1). In den letzten 30 Jahren (1990-2019) verdoppelte sich die durchschnittliche Anzahl der Hitzetage pro Jahr von 5 auf 10 Tage im Vergleich zum Zeitraum 1961-1990 (LUBW 2020).

Um mit diesen bereits eingetretenen und den noch zu erwartenden klimatischen Veränderungen bestmöglich umgehen zu können, besteht die dringende Notwendigkeit, geeignete Anpassungsstrategien zu entwickeln und umzusetzen (Muthers et al. 2017). Insbesondere Städte gelten als besonders vulnerabel für die Auswirkungen des Klimawandels, wie z.B. extreme Hitze oder Stürme (Moraci et al. 2018). Zahlreiche Haupt- und Großstädte, wie z.B. Stuttgart, Karlsruhe oder Mannheim verfügen deshalb bereits über Konzepte oder Maßnahmen für Klimaanpassung (Reckien et al. 2018). In der Mehrzahl der kleinen und mittelgroßen Kommunen, welche die Majorität der Bevölkerung Baden-Württembergs beheimaten (Destatis 2020), ist die kommunale Klimaanpassung jedoch keineswegs ausreichend entwickelt (Vetter et al. 2017, S. 328), was auf mögliche Barrieren oder Hindernisse für Klimaanpassung hindeutet.

Im internationalen Diskurs gibt es eine Vielzahl von Publikationen, welche Hindernisse für kommunale Klimaanpassung thematisieren (z. B. Measham et al. 2011; Clar et al. 2013; Lehmann et al. 2015). Trotzdem sind die Erfolgsfaktoren zu deren Überwindung bisher größtenteils unbestimmt (Clar und Steurer 2019) und die praktische Umsetzung bleibt hinter dem Möglichen zurück (Wamsler et al. 2020). Auf Deutschland bezogen, widmet sich der 2019 erschienene Teilbericht Wirkung der Deutschen Anpassungsstrategie für die Kommunen u. a. der Frage nach dem aktuellen Implementierungsstand der kommunalen Klimaanpassung. Der Bericht stellt eine Lücke zwischen dem bereitgestellten Wissen und der praktischen Umsetzung und Anwendung in den Kommunen heraus und attestiert einen erheblichen Handlungsbedarf in der Verstetigung kommunaler Klimaanpassungsprozesse (Hasse und Willen 2019). Angesichts der Dringlichkeit der Situation stellt sich die Frage, wie das vorhandene Wissen zielgerichteter in kommunale Organisationen integriert und mit konkreten Maß- nahmen agil, systematisch und fokussiert umgesetzt werden kann.

Ziel dieser Publikation ist es, am Beispiel des stark vom Klimawandel betroffenen Landes Baden-Württemberg retardierende Momente für wirksame Klimaanpassungsstrategien in kommunalen Organisationsstrukturen zu identifizieren und darauf aufbauend, geeignete Lösungen zur Überwindung der identifizierten Barrieren zu erarbeiten. Zur Beantwortung der Fragen Wo liegen die Hemmnisse für die effektive Integration von Klimaanpassungsmaßnahmen in Kommunen? (nachfolgend Fragestellung 1) und daran anknüpfend Welche Lösungsansätze tragen zur Überwindung dieser Hemmnisse bei? (nachfolgend Fragestellung 2) wurde ein landesweiter Workshop initiiert. Die Besonderheit dieser Veröffentlichung liegt darin, dass im Rahmen des Workshops die Klimaschutz- und Klimaanpassungsmanager*innen, also die Entscheider und praktischen Umsetzer, befragt wurden. Zusammen mit diesen konnten Hemmnisse formuliert und praxisorientierte und anwendbare Lösungen erarbeitet werden.

\section{Kommunale Klimaanpassung: Akteur*innen und Methode}

Positionen in kommunalen Verwaltungen, die sich hauptsächlich mit Fragen des Klimawandels befassen, sind die Klimaschutz- oder seltener Klimaanpassungsmanager*innen. In ihrer Rolle als Change Agents, entwickeln sie Visionen und initiieren Veränderungsprozesse mit großer Reichweite (Bauer und Hertle 2015). Zu dem OnlineWorkshop wurden die im Netzwerk der Landesenergieagentur Baden-Württemberg registrierten Klimaschutzmanager*innen oder die für die Bereiche Klimaschutz und Klimaanpassung zuständigen Mitarbeiter*innen der Kommunen des Landes Baden-Württemberg eingeladen. Da die Klimaschutzmanager*innen in Baden-Württemberg nicht nur auf Städteebene, sondern häufig interkommunal tätig sind, erstrecken sich deren Einfluss- und Zuständigkeitsbereiche auf Gemeindeverwaltungsverbände (Bieber 2021). Insgesamt 13 Teilnehmer*innen mit einem Zuständigkeitsbereich von insgesamt ca. 30 Städten und 64 Gemeinden nahmen an dem Workshop teil.

Für die strukturierte Generierung der Ideen, fiel die Auswahl der Erhebungsmethode auf das elektronische Brainstorming. Die Methode gilt als zeiteffizient (Maaravi et al. 2020) und ist zudem sehr etabliert. Für die vollständige Sammlung der Ideen reichten Brainstorming-Sitzungen (nachfolgend Befragungen) von jeweils etwa 30Minuten Dauer. An die Befragungen schlossen sich eine 45-minütige 
Abb. 1 Anzahl heißer Tage in Baden-Württemberg (Flächenmittel pro Jahr seit 1951), berechnet nach Daten des Deutschen Wetterdienstes (LUBW 2020)

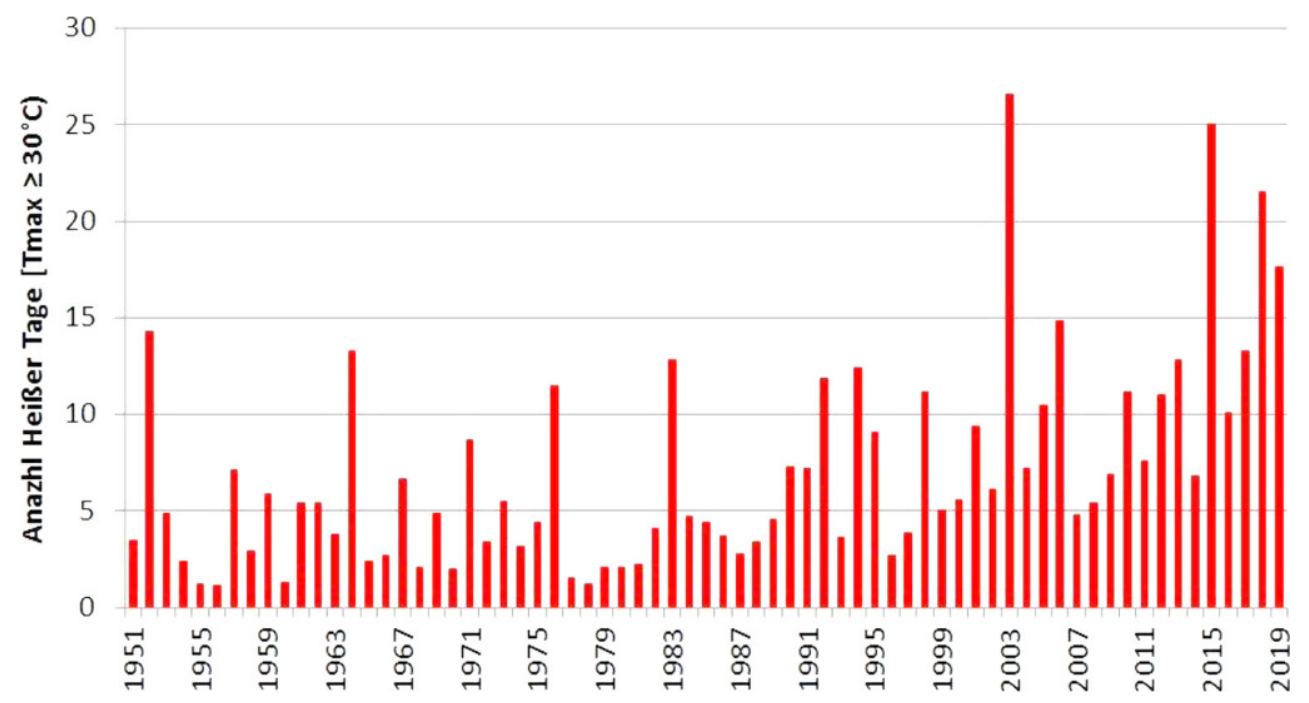

Diskussion aller Teilnehmer*innen sowie die Präsentation der Gesamtergebnisse beider Gruppen. Darauf aufbauend definierten die Teilnehmer*innen im Rahmen der induktiven Kategorienbildung nach Mayring (2010, S. 66) drei Oberbegriffe (politische Rahmenbedingungen, Kommunikation und Organisation) und ordneten die verschiedenen Ideen entsprechend $\mathrm{zu}$.

\section{Hemmnisse für die Integration von Klimaanpassungsmaßnahmen in Kommunen}

Die herausgestellten, wesentlichen Ursachen zur Beantwortung der Fragestellung 1 lauten wie folgt:

- Politische Rahmenbedingungen

Umwelt- und Klimathemen zählen zum Wahlprogramm der Koalitionsregierung und in der jüngsten Vergangenheit konnten durchaus positive politische Entwicklungen in den Sektoren Klima, Energie und Umweltschutz beobachtet werden. Im Mittelpunkt des politischen Handelns landen jedoch oft andere Kernthemen wie Wirtschaft, Beschäftigung und Mobilität, so dass dem Klimawandel bei der strategischen politischen Ausrichtung nicht immer der angemessene Stellenwert eingeräumt wird. Zudem fehlen in vielen Kommunen und Gemeinden häufig die personellen und zeitlichen Ressourcen, um zusätzliche und gezielte Mittel für Klimaanpassungsmaßnahmen zu akquirieren. Erwähnung fanden außerdem die Befürchtungen, dass neben der aktuellen, durch die CoronaPandemie verursachten Situation, zukünftig essenzielle Klimathemen seitens der Politik aufgeschoben werden könnten.
- Kommunikation

In der Kommunikation zwischen wichtigen StakeholderGruppen wie der Bürgerschaft oder des Stadt- bzw. Gemeinderats ist die Abstimmung in Themen wie Klimaschutz und Klimaanpassung von großer Bedeutung. Allerdings gibt es gegenüber Klimaschutzmaßnahmen, wie z. B. Windparks, zum Teil erhebliche Widerstände seitens der Bevölkerung, welche unter dem Begriff Nimby-Effekt (engl. „not in my backyard“) zusammengefasst werden. Da der Nimby-Effekt nicht rational ist (Pol et al. 2006), ist zu befürchten, dass auch gegen Klimaanpassungsmaßnahmen, welche z.B. die Parkplatzsituation (Entsiegelung) und die Mobilitätssituation (Erhalt anstatt Neubau von Straßen) tangieren (UMBW 2015), vermehrt Widerstände zu erwarten sind. Hinsichtlich der Kommunikation von Anpassungsmaßnahmen fehlt es kommunalen Akteur*innen zudem an Erfahrung. Die daraus resultierende Unsicherheit erschwert die Beschlussfassung pro Klimaanpassung.

- Organisation

Kommunale Einrichtungen sind i. A. hierarchisch und funktional gegliedert und hauptsächlich auf die Bearbeitung konkreter, tagesaktueller Abläufe und Aufgaben hin organisiert. Positionen wie die Klimaschutzmanager*in sind auf Verwaltungsebene häufig in Abteilungen wie der Stadtplanung, dem Grünflächenamt oder dem Umweltamt integriert und haben daher nur einen begrenzten Einfluss auf strategische Entscheidungen. Diese Einbettung erfolgt meist in einer sogenannten Linienorganisation und das hat zur Folge, dass Empfehlungen der Klimastellen in der Organisation oft nicht unmittelbar und durchschlagend wahrgenommen werden können und deren Arbeit daher eher reaktiv statt proaktiv wirken kann. 


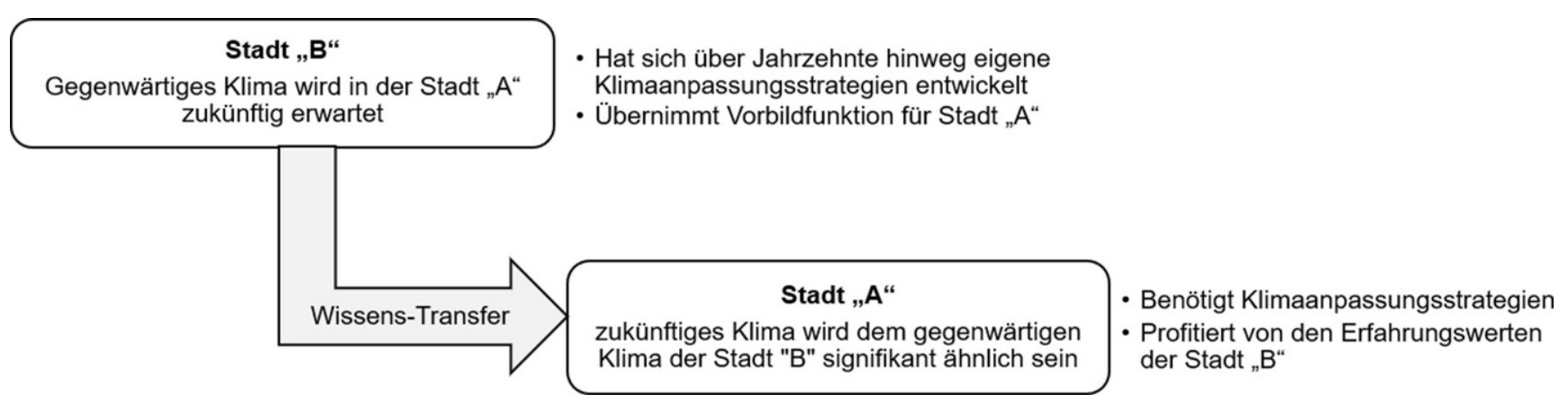

Abb. 2 Klimapartnerstädte-Systematik in Anlehnung an Rohat et al. (2017), eigene Darstellung

\section{Ableitung von Lösungsansätzen zur Überwindung der gefundenen Hemmnisse für kommunale Klimaanpassung}

Die Formulierung und Weiterentwicklung der gefundenen Lösungen fand im abschließenden moderierten Fokusgespräch mit den Teilnehmern statt. Die drei vorgeschlagenen Lösungsansätze sind im Folgenden.

- Lösungsansatz Netzwerke

Die Teilnehmer*innen schlugen lebendige Netzwerke als Teil eines möglichen Lösungsszenarios zur Überwindung von Hindernissen in den Bereichen Politik und Kommunikation vor. Ein Netzwerk aus Kommunen einer Region und lokalen Institutionen, wie beispielsweise Bildungseinrichtungen, Handwerkskammern oder Vereinen, könnte nicht nur eine deutlich bessere Sichtbarkeit bieten, sondern auch ein größeres Potenzial für den Austausch in Form von Klimainformationsprojekten oder für die gemeinsame Beantragung von Fördermitteln. Darüber hinaus könnten gemeinsame Workshops und Netzwerkveranstaltungen von Klimaschutz- und Klimaanpassungsmanager*innen aus mehreren Kommunen eine höhere gesellschaftliche und politische Wirkung haben als die Summe der jeweiligen lokalen Einzelaktivitäten. Sowohl ein besserer regionaler Wissenstransfer als auch ein Zusammenschluss von regionalen Bürgerinitiativen und politischen Akteur*innen könnten zudem die Akzeptanz und Wirkung lokaler Anpassungsmaßnahmen deutlich fördern und eine Abstimmung in der KlimaKommunikation zwischen Akteur*innen aus Kommune, Wirtschaft, Politik und Wissenschaft voranbringen.

- Lösungsansatz Wissenstransfer Zur Unterstützung in der kommunalen Klima-Kommunikation wurde zudem der Einbezug überregionaler Stakeholder-Gruppen wie Umweltverbänden oder Forschungsinstituten vorgeschlagen, um die Zielgruppe der Bürger*innen besser zu erreichen. $\mathrm{Zu}$ den möglichen Potenzialen dieses Austauschs gehören die Erstellung von Klimaprognosen, z.B. in Form von Hochwasserrisiko- karten, die Identifizierung von Hitzespots in städtischen Gebieten und die Sensibilisierung für die Notwendigkeit von Klimaanpassungsstrategien. Diese Maßnahmen könnten helfen, die Thematik der Klimaanpassung aus dem wissenschaftlich-abstrakten Raum heraus zu holen, zu einer greifbaren und bürgernah-kommunizierten Sichtbarkeit der Entwicklungen. Ein konkretes Lösungsszenario könnte auch im Konzept der sogenannten Klimapartnerstädte liegen. Rohat et al. (2017) definieren diese Städte als Städtepaare, bei welchen zu erwarten ist, dass das zukünftige Klima einer Stadt „A“ dem gegenwärtigen Klima einer anderen Stadt „B“ signifikant ähnlich sein wird (Abb. 2).

Durch Übertragung von Beispielen aus Klimapartnerstädten wird der Abstraktionsgrad verringert und die gesellschaftliche und politische Akzeptanz möglicher Klimafolgen erhöht. Dieses Konzept könnte zukünftig in noch größerem Rahmen auf deutsche Städte übertragen werden und einen Beitrag zur bürgernahen Klimakommunikation leisten.

- Lösungsansatz Organisation

In der Implementierung von Stabsstellen für Klimaschutz- und Klimaanpassungsmanager*innen liegen Potenziale für eine deutliche Erweiterung des Handlungsrahmens und für eine verbesserte Sichtbarkeit des Wirkens dieser Akteur*innen. Darüber hinaus könnte ein Zusammenschluss der Klimaschutz- und Klimaanpassungsstellen mehrerer Kommunen einer Region die Integration von Anpassungsmaßnahmen effektiver voranbringen. Aus der Vernetzung mit anderen Klimastellen auf Landes-, Leitungs-, und Ressortebene könnte darüber hinaus ein Verbund handlungsfähiger Akteur*innen entstehen. 


\section{Transformative Klimaanpassungs-Netzwerke}

Vor allem in der strategischen Unterstützung der Kommunen durch Stakeholder könnte zukünftig ein wichtiger Schlüssel zum Gelingen von Klimaanpassungsmaßnahmen liegen (Ebermann 2020, S. 28). Einige aktuelle Forschungsvorhaben beschäftigen sich mit der Frage, in welcher Form Netzwerke unterschiedlicher Stakeholder die notwendige Unterstützung für kommunale Klimaanpassung leisten könnten.

Ein Beispiel für ein laufendes Forschungsprojekt mit Netzwerk-Ansatz ist das EU-Life-Projekt Evolving Regions der Technischen Universität Dortmund. Dieses zielt darauf ab, acht Partnerregionen in Nordrhein-Westfalen und der Niederlande klimarobust zu gestalten. Dazu werden Stakeholder dieser ländlich geprägten Regionen von der Technischen Universität Dortmund und fünf Partnerinstitutionen mit übergreifenden Dialogformaten und individuell zugeschnittenen Serviceleistungen unterstützt. Die Ergebnisse werden in einem neuartigen Konzept aus passgenauen Roadmaps für Klimaanpassung zusammengefasst (Schultze 2020).

Erfolgspotenziale für die Zukunft könnten in der Erweiterung von Netzwerk-Projekten um Aspekte des Wissenstransfers und der Einbindung von Klimapartnerstädten liegen. Durch die visuelle Darstellung einer möglichen Klimazukunft könnten die beteiligten Akteur*innen einer Projektregion für die Auswirkungen des Klimawandels sensibilisiert werden. Für die Verstetigung der Projektergebnisse wäre zudem zu empfehlen, auch die organisatorischen Hindernisse hinsichtlich der Positionierung von Klimastellen in Kommunen im Prozess zu betrachten.

\section{Zusammenfassung und Ausblick}

Der im Rahmen dieser Veröffentlichung beschriebene Workshop identifizierte die akuten Barrieren für die Umsetzung von Klimaanpassungsstrategien in kommunalen Organisationsstrukturen. Die Klimaschutz- und Klimaanpassungsmanager*innen sind verantwortliche Change Agents mit z.T. großen Einfluss- und Zuständigkeitsbereichen. Jedoch sind sie in vielen Fällen nicht mit den notwendigen Mitteln ausgestattet, um ihrer Aufgabe hinreichend gerecht zu werden. Seien es mangelnde personelle und finanzielle Ressourcen, die fehlende Unterstützung und Kooperation durch wichtige Stakeholder oder der angemessene politische Beistand. Diese retardierenden Barrieren wurden im nächsten Schritt adressiert, um Lösungsansätze zur Überwindung dieser Hemmnisse zu finden.

Zunächst wird ein Zusammenschluss verschiedener Akteur*innen einer Region zu lebendigen Netzwerken für Klimaanpassung empfohlen, um die Initiierung und Umset- zung gemeinsamer Projekte sowie die Akzeptanz und Sichtbarkeit von Maßnahmen zu fördern. Begleitend könnte ein verbesserter Wissenstransfer durch ausgewählte Stakeholder-Kooperationen die Unsicherheiten in Bezug auf Klimaauswirkungen anhand von verständlichen Beispielen auflösen. Zuletzt zeigte der Workshop, dass die Positionierung der Klima-Stellen in der kommunalen Organisation einen direkten Einfluss auf ihre Einflussmöglichkeiten und $\mathrm{Au}-$ Benwirkung hat. Potenziale liegen z. B. in der Implementierung von Klima-Stabsstellen sowie in der besseren Vernetzung der Klimastellen auf sämtlichen Hierarchieebenen.

Zusammengenommen könnten die Ergebnisse dieser Veröffentlichung zu mehr Kohärenz in der kommunalen Anpassungsforschung beitragen und dabei helfen, eine Wissensbasis für die Integration von Klimaanpassung in kommunalen Strukturen aufzubauen. Da sich Klimaanpassungsprozesse nicht nachhaltig top-down delegieren lassen (Baasch et al. 2012), ist es wichtig, die Akteur*innen an der Basis zu befähigen und ihnen die richtigen Instrumente zum Handeln an die Hand zu geben. Dabei ist der Dialog zwischen Stakeholdern und den kommunalen Akteur*innen vor Ort essenziell, um eine nachhaltige Systematik für die erfolgreiche und agile Initiierung und Verstetigung von kommunaler Klimaanpassungsmaßnahmen sicherzustellen.

Funding Open Access funding enabled and organized by Projekt DEAL.

Open Access Dieser Artikel wird unter der Creative Commons Namensnennung 4.0 International Lizenz veröffentlicht, welche die Nutzung, Vervielfältigung, Bearbeitung, Verbreitung und Wiedergabe in jeglichem Medium und Format erlaubt, sofern Sie den/die ursprünglichen Autor(en) und die Quelle ordnungsgemäß nennen, einen Link zur Creative Commons Lizenz beifügen und angeben, ob Änderungen vorgenommen wurden.

Die in diesem Artikel enthaltenen Bilder und sonstiges Drittmaterial unterliegen ebenfalls der genannten Creative Commons Lizenz, sofern sich aus der Abbildungslegende nichts anderes ergibt. Sofern das betreffende Material nicht unter der genannten Creative Commons Lizenz steht und die betreffende Handlung nicht nach gesetzlichen Vorschriften erlaubt ist, ist für die oben aufgeführten Weiterverwendungen des Materials die Einwilligung des jeweiligen Rechteinhabers einzuholen.

Weitere Details zur Lizenz entnehmen Sie bitte der Lizenzinformation auf http://creativecommons.org/licenses/by/4.0/deed.de.

\section{Literatur}

Baasch S, Bauriedl S, Hafner S, Weidlich S (2012) Klimaanpassung auf regionaler Ebene: Herausforderungen einer regionalen Klimawandel-Governance. RuR Spatial Res Plan 70(3):191-201. https://doi.org/10.1007/s13147-012-0155-1

Bauer H, Hertle H (2015) Akteure des Wandels. Ökol Wirtsch 30(2):23-24. https://doi.org/10.14512/OEW300223

Bieber H (2021) Klimaschutzmanager. KEA-BW. https://www.keabw.de/kommunaler-klimaschutz/netzwerk/klimaschutzmanager. Zugegriffen: 5. März 2021

Clar C, Steurer R (2019) Climate change adaptation at different levels of government: characteristics and conditions of policy change. 
Nat Resour Forum 43(2):121-131. https://doi.org/10.1111/14778947.12168

Clar C, Prutsch A, Steurer R (2013) Barriers and guidelines for public policies on climate change adaptation: a missed opportunity of scientific knowledge-brokerage. Nat Resour Forum 37(1):1-18. https://doi.org/10.1111/1477-8947.12013

Destatis (2020) Kreisfreie Städte und Landkreise am 31.12.2019. https://www.destatis.de/DE/Themen/Laender-Regionen/Regional es/Gemeindeverzeichnis/Administrativ/04-kreise.html. Zugegriffen: 5. März 2021

Ebermann V (2020) Herausforderungen der Strategieentwicklung. In: Entwicklung von staatlichen Strategien zur Klimaanpassung. Springer VS, Wiesbaden, S 18-39

Hasse J, Willen L (2019) Umfrage Wirkung der DAS für die Kommunen. Teilbericht. https://www.umweltbundesamt.de/sites/default/ files/medien/1410/publikationen/2019-01-21_cc_01-2019_umfra ge-das.pdf. Zugegriffen: 4. März 2021

Lehmann P, Brenck M, Gebhardt O et al (2015) Barriers and opportunities for urban adaptation planning: analytical framework and evidence from cities in Latin America and Germany. Mitig Adapt Strateg Glob Change 20:75-97. https://doi.org/10.1007/s11027013-9480-0

LUBW (2020) Kurzfassung Klimabilanz 2019. https://pudi.lubw.de/ detailseite/-/publication/10102. Zugegriffen: 5. März 2021

Maaravi Y, Heller B, Shoham Y et al (2020) Ideation in the digital age: literature review and integrative model for electronic brainstorming. Rev Manag Sci. https://doi.org/10.1007/s11846-02000400-5

Mayring P (2010) Qualitative Inhaltsanalyse - Grundlagen und Techniken, 11. Aufl. Beltz, Weinheim, Basel

Measham TG, Preston BL, Smith TF et al (2011) Adapting to climate change through local municipal planning: barriers and challenges. Mitig Adapt Strateg Glob Change 16:889-909. https://doi.org/10. 1007/s11027-011-9301-2

Moraci F, Errigo MF, Fazia C, Burgio G, Foresta S (2018) Making less vulnerable cities: resilience as a new paradigm of smart planning. Sustainability 10(3):755. https://doi.org/10.3390/su10030755
Muthers S, Laschewski G, Matzarakis A (2017) The summers 2003 and 2015 in South-West Germany: heat waves and heat-related mortality in the context of climate change. Atmosphere 8(11):224. https://doi.org/10.3390/atmos8110224

Pol E, Di Masso A, Castrechini A, Bonet M, Vidal T (2006) Psychological parameters to understand and manage the NIMBY effect. Eur Rev Appl Psychol 56(1):43-51. https://doi.org/10.1016/ j.erap.2005.02.009

Reckien D, Salvia M, Heidrich O, Church JM, Pietrapertosa F, De Gregorio-Hurtado $\mathrm{S}$ et al (2018) How are cities planning to respond to climate change? Assessment of local climate plans from 885 cities in the EU-28. J Clean Prod 191:207-219. https://doi.org/10. 1016/j.jclepro.2018.03.220

Rohat G, Goyette S, Flacke J (2017) Twin climate cities-an exploratory study of their potential use for awareness-raising and urban adaptation. Mitig Adapt Strateg Glob Change 22:929-945. https:// doi.org/10.1007/s11027-016-9708-x

Schultze J (2020) Evolving regions. https://evolvingregions.com/. Zugegriffen: 8. März 2021

Stölzle M, Stahl K (2011) Wassernutzung und Trockenheitsindikatoren in Baden-Württemberg. Standort 35(3):94. https://doi.org/10. 1007/s00548-011-0169-x

UMBW (2015) Anpassungsstrategie BWB. https://um.baden-wuerttem berg.de/fileadmin/redaktion/m-um/intern/Dateien/Dokumente/4 Klima/Klimawandel/Anpassungsstrategie.pdf. Zugegriffen: 5 . März 2021

Vetter A, Chrischilles E, Eisenack K et al (2017) Anpassung an den Klimawandel als neues Politikfeld. In: Brasseur G, Jacob D, Schuck-Zoeller S (Hrsg) Klimawandel in Deutschland. Springer Spektrum, Berlin, Heidelberg, S 325-334

Wamsler C, Wickenberg B, Hanson H et al (2020) Environmental and climate policy integration: Targeted strategies for overcoming barriers to nature-based solutions and climate change adaptation. J Clean Prod 247:119-154. https://doi.org/10.1016/j.jclepro. 2019.119154 